\title{
Is it turquoise + fuchsia $=$ purple or is it turquoise + fuchsia $=$ blue?
}

\section{Giordano Beretta, Nathan Moroney}

Giordano B. Beretta, Nathan M. Moroney, "Is it turquoise + fuchsia = purple or is it turquoise + fuchsia = blue?," Proc. SPIE 7866, Color Imaging XVI: Displaying, Processing, Hardcopy, and Applications, $78660 \mathrm{H}$ (25 January 2011); doi: 10.1117/12.872581

Event: IS\&T/SPIE Electronic Imaging, 2011, San Francisco Airport, California, United States 


\title{
Is it turquoise + fuchsia $=$ purple or is it turquoise + fuchsia $=$ blue?
}

\author{
Giordano B. Beretta, Nathan M. Moroney \\ Hewlett-Packard Laboratories, Print Production Automation Lab \\ 1501 Page Mill Road, Palo Alto, USA
}

\begin{abstract}
The first step in communicating color is to name it. The second step is color semiotics. The third step is introducing structure in the set of colors. In color education at all levels, this structure often takes the form of formulæ, like red + green $=$ yellow, or turquoise + red $=$ black. In recent times, Johannes Itten's color theory and its associated color wheel have been very influential, mostly through its impact on Bauhaus, although a number of color order systems and circles have been introduced over the centuries.

Students get confused when they are trying to formulate the color name arithmetic using the structure of color order systems and concepts like complementary colors and opponent colors. Suddenly turquoise + fuchsia = purple instead of blue; purple and violet become blurred, and finally the student's head explodes under the epistemological pressures of Itten, Albers, Goethe, Runge, Newton, da Vinci, and all the other monsters of color structure.

In this contribution we propose a systematic presentation of structure in color, from color theories to color naming. We start from the concept of color perception introduced by da Vinci and work ourselves through color measurement, color formation, and color naming, to develop the basis for a robust system based on table lookup and interpolation.

One source of confusion is that color naming has been quite loose in color theory, where for example red can be used interchangeably with fuchsia, and blue with turquoise. Furthermore, common color terms are intermingled with technical colorant terms, for example cyan and aqua or fuchsia and magenta. We present the evolution of a few color terms, some of which have experienced a radical transition over the centuries, and describe an experiment showing the robustness of crowd-sourcing for color naming.
\end{abstract}

Keywords: color names; color theories; crowd-sourcing; education

\section{COLOR THEORIES}

Humans have used color symbolism at least since the paintings in the Qafzeh Cave around 92,000 B.C.E. ${ }^{1}$ and relations among colors have been described at least since 800 B.C.E. when the Upanishad treatises were written in India. While industrial color scientists are mostly concerned with single colors, often considered in aperture mode, in the pictorial arts complex color and color palettes are more important.

A painter who "has made it" usually has a fixed palette and for a specific painting will maybe add a single colorant to his palette (see Fig. 1). Hence, it is not that a painter picks a subject, determines the colorants, and starts painting it. For a pictorial artist, the palette is an intimate and relatively static entity.

However, painters reach this state only after "having seen the light" and having found their style; beforehand, they work hard to develop their tacit knowledge, which includes developing an intuition on how to organize their colors and developing recipes for the colors they want to create. In this phase of their development, which requires extensive experimentation with many colorants, artists encounter the necessity to be able to organize their colors systematically, so they can easily categorize and retrieve them. In this paper, we build the body

Further author information: (Send correspondence to N.M.M.)

G.B.B.: E-mail: giordano.beretta@hp.com, Telephone: +1 6508576713

N.M.M.: E-mail: nathan.moroney@hp.com, Telephone: +1 6502362825

Blog: mostlycolor.ch

Color Imaging XVI: Displaying, Processing, Hardcopy, and Applications, edited by Reiner Eschbach,

Gabriel G. Marcu, Alessandro Rizzi, Proc. of SPIE-IS\&T Electronic Imaging, SPIE Vol. 7866

$78660 \mathrm{H} \cdot$ · C 2011 SPIE-IS\&T · CCC code: 0277-786X/11/\$18 doi: 10.1117/12.872581 


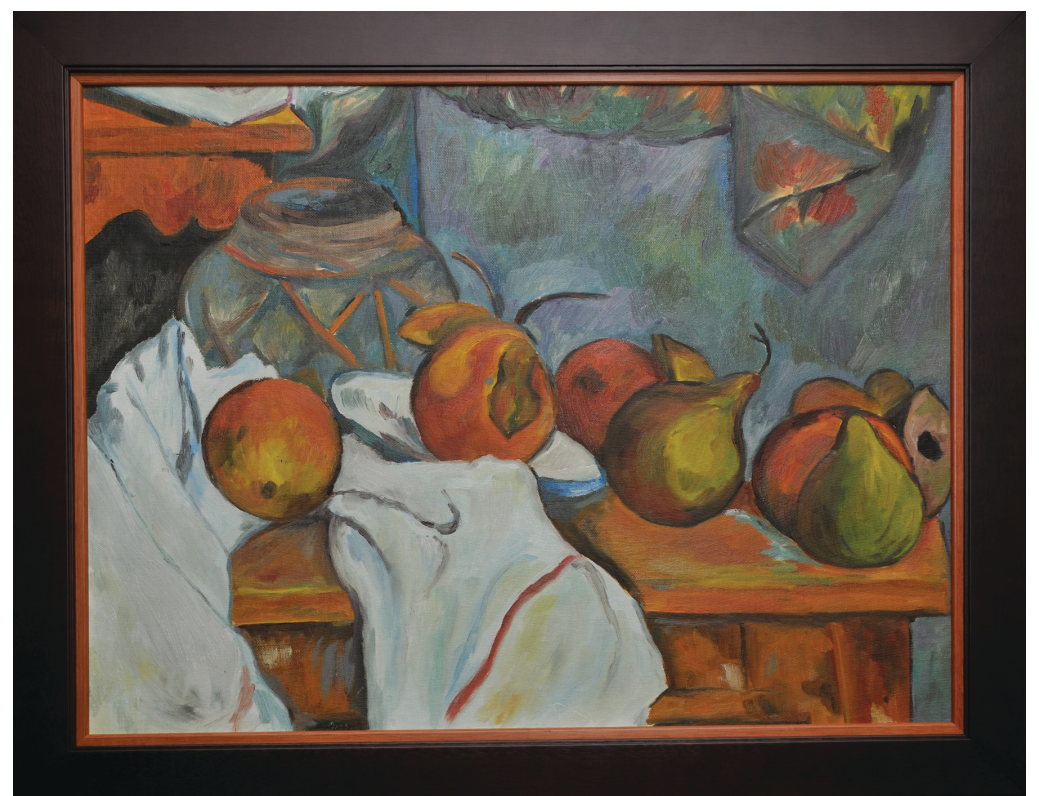

Figure 1. Technical books for artists allow the replication of famous artist's color palettes. Still life in Paul Cézanne's style, by Yoko Nonaka.

of knowledge necessary to understand how to build such color systems, without entering into the details of the systems themselves, which has been done by others. ${ }^{2}$

As mentioned at the beginning, this is a very old problem. In the western culture it made significant progress during the Renaissance with Leonardo da Vinci, who contributed six radically new concepts:

- color is a perceived entity

- we can construct a color order system

- black \& white are colors*

- there are 3 pairs of opponent colors (black-white, red-green, yellow-blue)

- simultaneous contrast: opponent colors mutually reinforce ${ }^{\dagger}$

- color filter arrays (spectroradiometry) can be used to determine color mixtures ${ }^{\ddagger}$

* "De' semplici colori, il primo è il bianco, benché i filosofi non accettano, nè il bianco, nè il nero nel numero de' colori, perche l'uno è causa de' colori, e l'altro è privazione" [3, chapter CLXI].

†'In chapter CLX: "Dei campi delle figure, cioè la chiara nell'oscuro, e l'oscura nel campo chiaro, del bianco col nero, o nero col bianco, pare più potente l'uno per l'altro, e così li contrarj l'uno per l'altro si mostrano sempre più potenti."

In chapter CLXII: "Delli colori di egual perfezzione, quello si dimostrerà di maggior eccellenza che sia veduto in compagnia del color retto contrario, \& il pallido col rosso, il nero col bianco, benche nè l'uno, nè l'altro sia colore: azzurro, e giallo, verde, e rosso, perché ogni colore si conosce meglio nel suo contrario, che nel suo simile, come l'oscuro nel chiaro, il chiaro nell'oscuro". ${ }^{3}$

¥‘Se vuoi con brevità vedere la varietà di tutti li colori composti, togli vetri coloriti, e per quelli guarda tutt’i colori della campagna, che doppo quello si veggono, e così vedrai tutti li colori delle cose, che doppo tal vetro si veggono essere tutte miste col color del predetto vetro, e vedrai quale sia il colore, che con tal mistione s'acconci, o guasti: se sarà il predetto vetro di color giallo, dico che la specie degl'obbietti, che per esso passano all'occhio, possono così peggiorare, come megliorare: e questo peggioramento in tal colore di vetro accaderà all'azzurro, e nero, e bianco sopra tutti gl'altri, e così anderai scorrendo con l'occhio le mistioni de' colori, le quali sono infinite: \& a quello modo farai elezzione di nuove invenzioni di colori misti, e composti, \& il medesimo si farà con due vetri di varj colori anteposti all'occhio, e così per te potrai seguitare" [3, chapter CLXI]. 
The concept of interest in this paper is that of three orthogonal axes of opponent color, shown in Fig. 2 in three different projections:
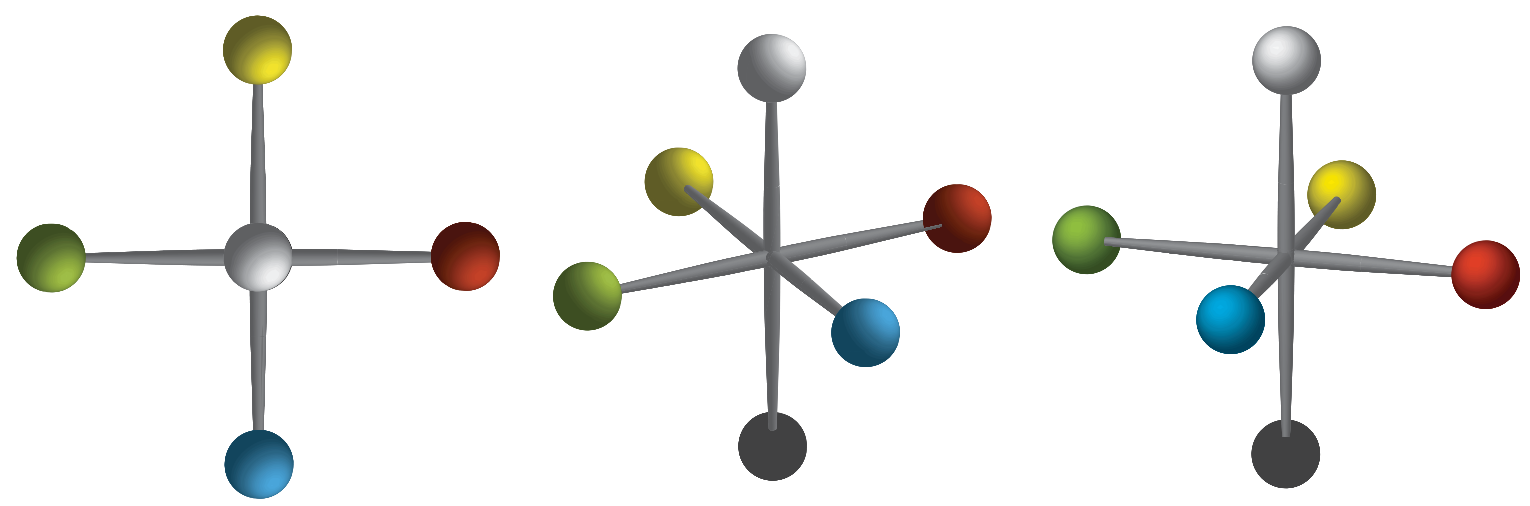

Figure 2. Leonardo da Vinci's opponent colors, rendered here using his chiaro-scuro technique to suggest 3-dimensionality. Left: view from top. Center: view from slight left. Right: view from slight right.

Leonardo's orthogonal axes are white-black, red-green, and yellow-blue. His new insight is that this model is based on the assumption that color is perceived, i.e., an abstract mental concept as opposed to a physical property related to light, colorants, or materials.

Note that in these three pairs the opponent colors are mutually exclusive, in the sense that there is no blackish white, greenish red, nor bluish yellow. Such a mutual exclusion is not experienced for other color pairs, and we can easily imagine colors like a yellowish green, a bluish red, or a reddish black.

Indeed, we can construct a 3-dimensional atlas of all colors with a bisection process. We start with Leonardo's axes of Fig. 2 and for a new color we place it in relation of the other colors. For example, we would place a brown between a red and a yellow, a little towards black, while we would put an orange more towards white, a little over a brown in the right view in Fig. 2.

We can imagine to start from three orthogonal axes with the cardinal colors, then, in each quadrant we find the intermediate color and keep bisecting the space in this fashion. If we perform two iterations and take the plan projection (left view in Fig. 2), and keep just the points on the gamut limit, we obtain the circle shown in Fig. 3 on the left side.

We can keep iterating until we obtain a continuous circle. Note that this construction is strictly abstract, independent of any physical realization of colors. Note also that we used the indeterminate grammar when naming the colors: a brown instead of brown or bluish instead of blue. We mention this because today the best known color circle is the one proposed by Johannes Itten of the Bauhaus movement (he called it Farbkreis [4, p. $22]$ ) and which is constructed in a completely different way, as shown in Fig. 4.

In Itten's Fablehre or color theory, two diametrically opposite colors are called direct complements (compare to Leonardo's opponent colors based on perception), while the colors on opposite vertices of a circumscribed equilateral triangle are called triadic complements. The colors adjacent to a given color are called harmonious colors. It is relatively straightforward to create a color selection tool blending opponent color theory with Itten's Farblehre. ${ }^{5}$

When the right sides of Figs. 3 and 4 are compared, it becomes clear that the color circle's structure is not universal, but depends on the chosen construction paradigm. In the first case the construction is based on perception while in the second it is based on language.

After Leonardo da Vinci, several artists and physicists have constructed color order systems following similar procedures. Of particular importance for color science is the work by the American Optical Society OSA, which has constructed an important color atlas in the following way. ${ }^{6}$ 

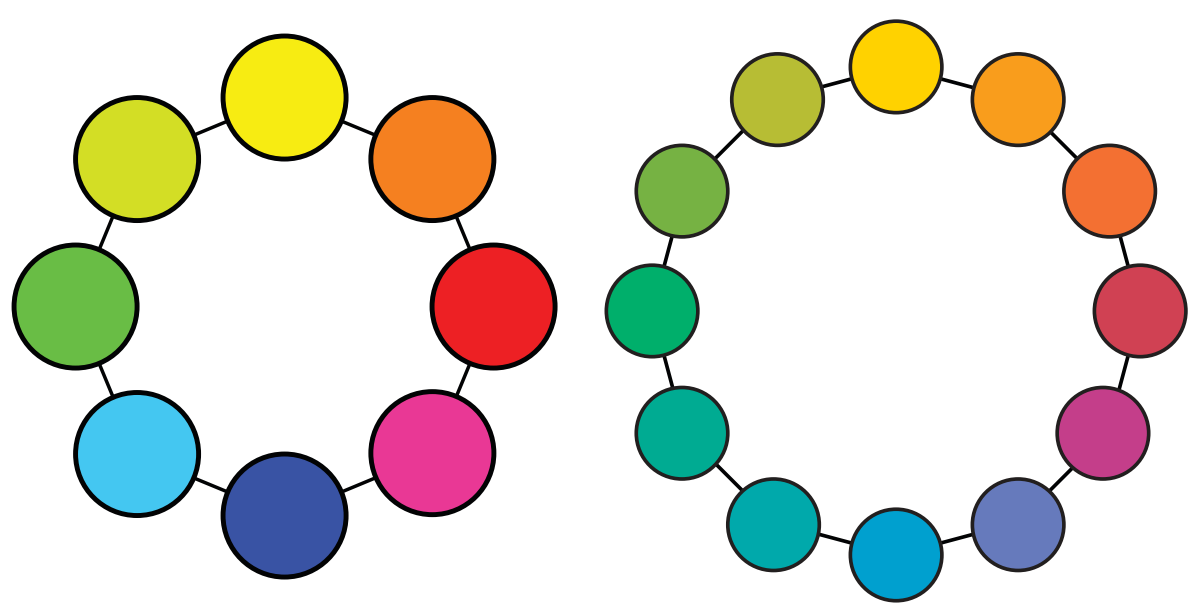

Figure 3. Left: Color circle obtained by projecting the left view in Fig. 2 and bisecting the quadrants. Right: Corresponding color circle with 12 subdivisions. The colors are different because of the in the left view we chose samples of maximal vividness on your display monitor regardless of lightness, while on the right we smoothened the lightness variation while keeping the vividness on the NCS gamut. Therefore, for a given color theory, the color appearance depends on how the color circle is rendered.
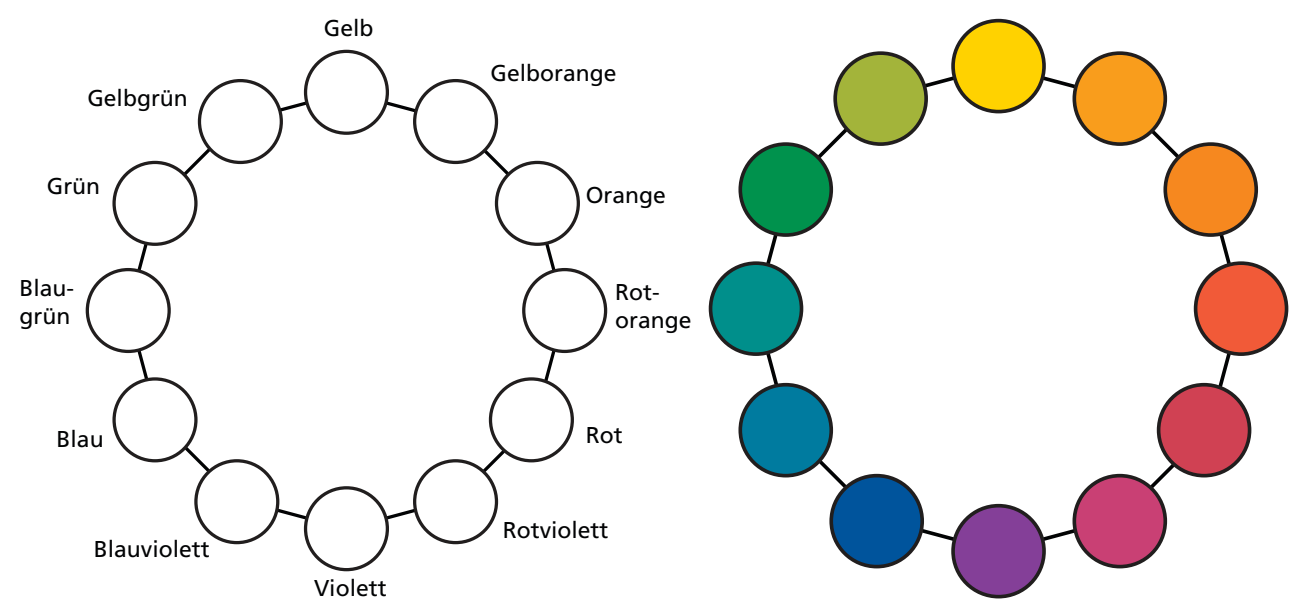

Figure 4. Left: Itten's Farbkreis is constructed by placing six monolexemic primary colors on a circle. In a second step a bilexemic secondary color is placed between each consecutive pair of primary colors. Right: A colored rendering based on the NCS color atlas.

The car paint division of Dupont created a large set of different colorants on small cardboard swatches (about $5 \mathrm{~cm}$ on each side). The set was then circulated among the color science luminaries of the time, which ordered them in three dimensions on an octahedral lattice. Eventually, they discarded samples where the perceived difference was below threshold, which left them with a completely uniform atlas, i.e., the difference distance between any two samples is proportional to the Euclidean distance in the atlas, independently of the colors.

The OSA atlas consists of pages that are parallel planes in the 3-dimensional space. Unfortunately this makes the atlas difficult to use in practice. For designers it is more useful to organize the set of samples like orange wedges. Each wedge can be reproduced as atlas pages, so browsing the atlas one traverses all hues in the color circle. Fig. 5 shows a page from Antal Nemcsics' Coloroid atlas (which is not based on the OSA system). ${ }^{7}$

\section{MEASURING COLOR}

Now that we know how to structure the set of all colors, ordering them and introducing relations like complementarity, harmony, etc., we have to find a way to measure color. This allows us to communicate color specifications 


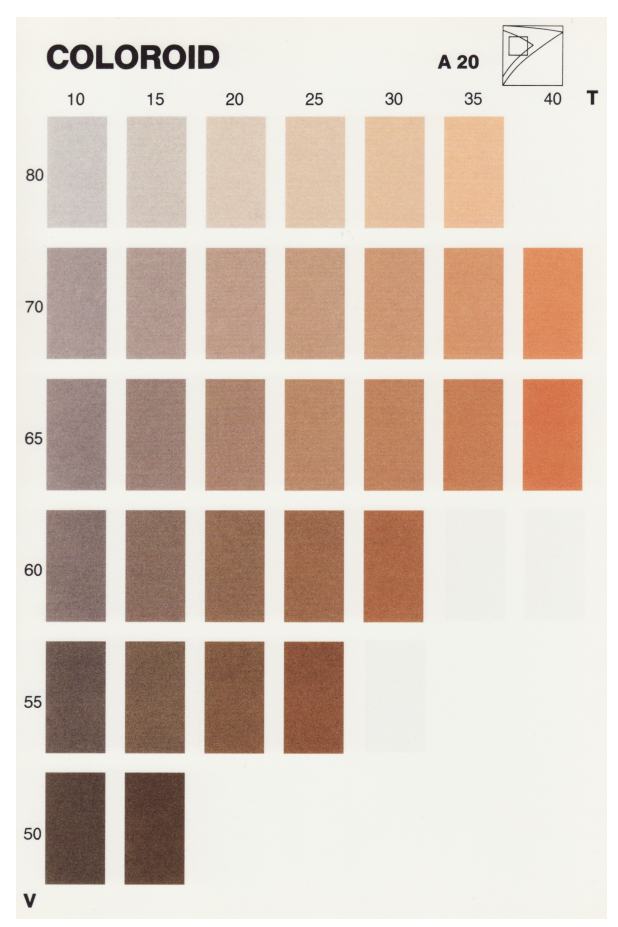

Figure 5. Page A20 in the Coloroid atlas.

without having to mail samples, and allows us also to verify the adherence to tolerances.

When we have a color atlas, all we need to do is introduce a coordinate system. For a space like OSA where the samples are on a lattice, these are cartesian coordinates. When the atlas is structured as wedges or leaves, like in the Coloroid system, cylindrical coordinates are the natural system.

For example, in Fig. 5 the first coordinate is the angle $A$ which is the page. The second coordinate is the abscissa $T$, and the third the ordinate $V$. When we need to communicate a color, we look up the closest sample in the atlas and then transmit three numbers, for example $(A, T, V)=(20,25,60)$. The receivers consult their Coloroid atlas to see this color's sample.

Similarly, a tanner who has to ensure that all leather swatches making up a handbag have the same color, let us say $(A, T, V)=(20,25,60)$, can verify that each swatch is more similar to this sample than to any of the 26 adjacent samples in the atlas (eight on the same page and nine each on the preceding and following pages).

Although this method is economical, it is not very useful when we have to characterize many color samples or when the tolerances are very tight. For example, in the textile industry the color of a roll of cloth for suits has to be completely uniform (i.e., below visual threshold), hence the dyeworks needs to continuously monitor the cloth exiting the dye bath. We would like to perform a physical measurement instead of doing a visual match.

Unfortunately, this is much harder than it might appear on a cursory look, because color is not a physical phenomenon but an optical illusion taking place in the human visual system (HVS). The best we can hope for, is to find an experiment producing a quantity that is well correlated with what we perceive. In other words, because we are not able to build a physiological model of color perception, we envision a psychophysical experiment in which we study the behavioral reaction elicited by a physical stimulation.

The solution is an experiment called color matching and is described conceptually with the apparatus shown in Fig. 6. It is a black box with an aperture or window in the front to look inside. On the opposing wall there is a perfectly white screen, halved by a black partition protruding towards the window. On the left side, the pinkish bulb represents a light source whose color we would like to characterize. On the right side there are three light sources we call primaries. 


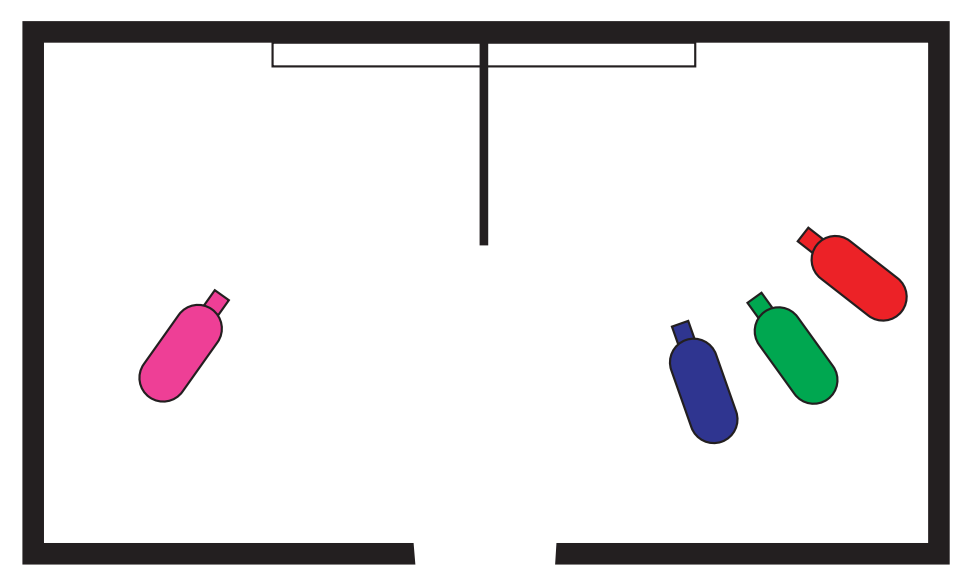

Figure 6. Conceptual rendering of a box for side-by-side color matching experiments. Alternate experimental set-ups are to flicker the two source groups on a single field, or to show each half of the bipartite field to a different eye for haploscopic color matching.

The particular colors of the three primaries are not important, they just have to be linearly independent, because as we saw in the previous section, we need a 3-dimensional system to specify color. The method consists in adjusting the photon flux output of the three primary sources until the two halves of the screen match. Fig. 7 illustrates the mechanics of the experiment.
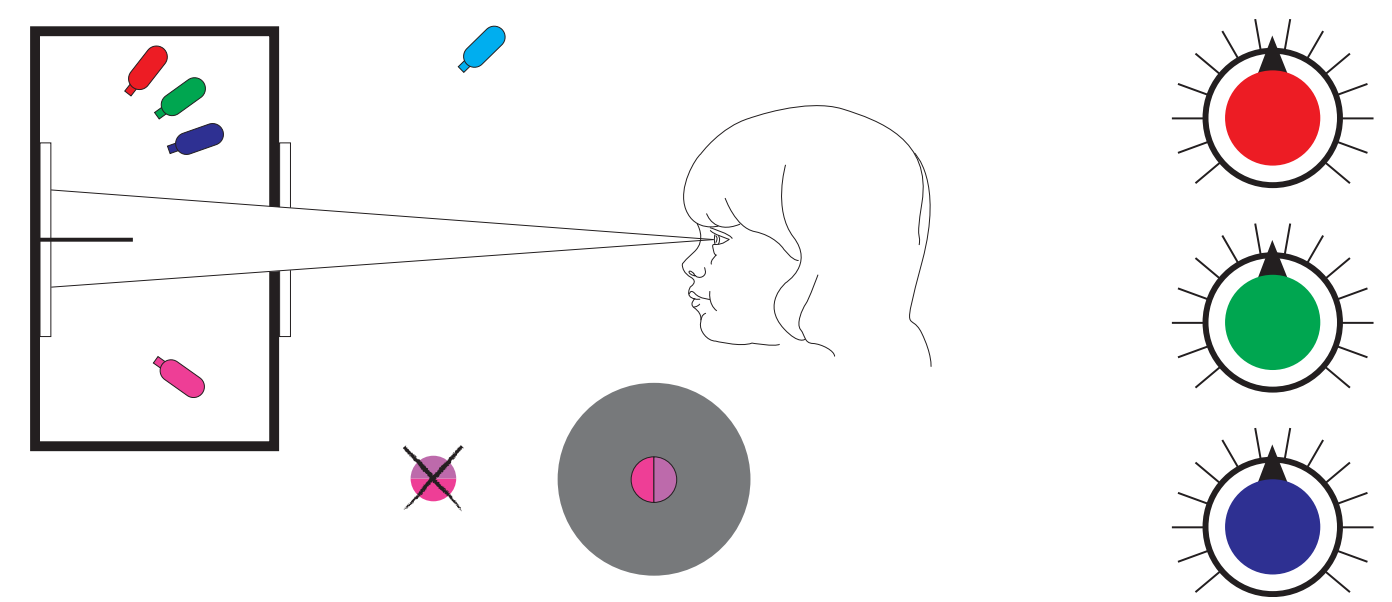

Figure 7. Elements and steps of the color matching experiments. The three knobs at right are turned until the two halves of the bipartite field match. If a match cannot be achieved, a primary source is moved to the other side to desaturate the test color; the corresponding knob is given a minus sign. The optional light source outside the box can be used to create a surround for adaptation.

The observer looks at the screen through the aperture and sees two half-circles as show in the inset (note that the elements in the figure are shown from different viewpoints). The observer has three knobs, as shown on the right side. Each knob controls the photon flux of the primary source of corresponding color. Around each knob is an arbitrary linear scale. The observer turns the knobs (stimulus control) until the two semi-circles match in color (behavioral reaction). At this point, the three numbers on the scales of the knobs are the coordinates specifying the color on the left side.

The color formation in the box is linear and the HVS is more or less linear, therefore the values on the scales are not important: all is needed to map from one box to another, is to apply a linear transform. This is also the reason the particular primary sources chosen are not important. If we want to change the light sources, all 
we have to do is to conduct the experiment with each of the old light sources in the left side of the box and the new light sources in the right side. The three coordinate triplets are the columns of a matrix that transforms the coordinates from the old to the new system.

When the color in the left side is so vivid that it cannot be matched, the trick is to desaturate this color by moving from the right to the left side the light source of the opposite color. On the scale of the knob corresponding to the moved light source, the sign is changed from positive to negative.

The last detail in Fig. 7 is the ambient light. Around the aperture there is a second screen illuminated with the external light source indicated with the light blue bulb.

Let us now focus on the physics. Newton had discovered that the color of a light source changes with its spectral power distribution, where the latter is defined as the power (in Watts per meter) of the radiation at each wavelength of the visible gamut.

If in the left side of the box in Fig. 7 we deploy a monochromatic light source-for example shining a white light through a prism (Newton's method) or through a diffraction grating (Grimaldi's method) - we find the visual system's response at every wavelength. Of course, the HVS changes from person to person, so we have to repeat the experiment with many subjects and average the results to obtain a standard observer.

The values on the knobs for this standard observer yield the color matching functions shown in Fig. 8. The negative lobes simply come from having had to move a light source from the right to the left to desaturate the light at those wavelengths. This leads to the following procedure to specify object colors through an experiment:

1. use a spectroradiometer to measure the object's spectral distribution

2. integrate the spectrum using each of the color matching functions to obtain the tristimulus values

$$
\begin{aligned}
R & =k \int P_{\lambda} \bar{r}(\lambda) d \lambda \\
G & =k \int P_{\lambda} \bar{g}(\lambda) d \lambda \\
B & =k \int P_{\lambda} \bar{b}(\lambda) d \lambda
\end{aligned}
$$

Note that we have computed integrals, hence the spectrum's shape is not important, just the area under the curve. This is the fundamental property called metamerism that makes color reproduction practical: we do not need to reproduce spectra, just the tristimulus values.

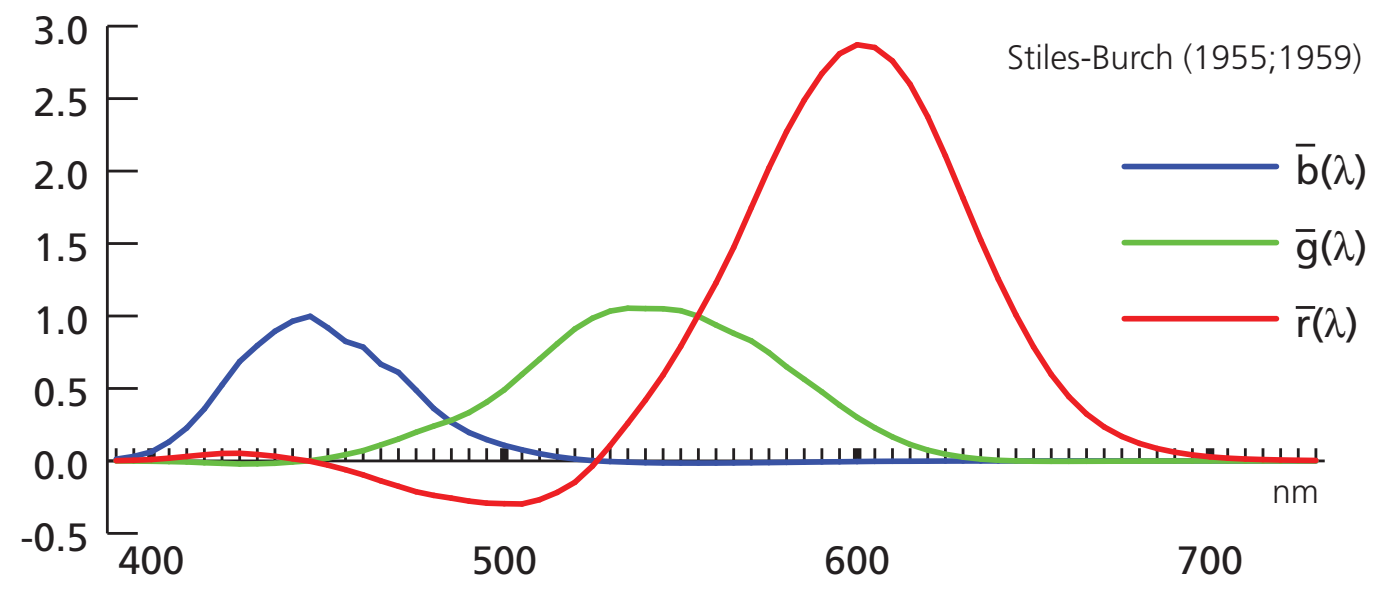

Figure 8. The color matching functions. 


\section{FORMING COLOR}

In Section 1 on color theories we saw that Leonardo wrote color is a perceived entity, and therefore to develop structures to describe color we have to abstract from its physical nature. However, when we want to create color, we need to develop techniques for physically realizing color. Since we have seen that to reproduce color we only need a trichromatic process, we are interested in developing methods to elicit color using a small number of primaries.

In the color matching experiment in Section 2 we used reddish, greenish, and bluish lights to match colors. This is the additive method, which has been known at least for 4000 years, since the invention of the potter's wheel, as described empirically by the Alexandrine Claudius Ptolomæus ( $100-170$ C.E. $)$ in his Optics, and scientifically by the Iraqi Abu Ali al-Hasan ibn al-Haytham $(965-1039)$ in his Book of Optics, ${ }^{\S}$ who became very well known in Europe under his Latinized name Alhazen. ${ }^{8}$

Returning to the color matching experiment, instead of an aperture color formed by a light source uniformly filling the visual field, we use three spot lights shining on a screen in a dark room, as shown on the left in Fig. 9. The spot lights are moved so that the light cones are not superimposed and we can see the illustrated color effects when the three light sources are at full power.
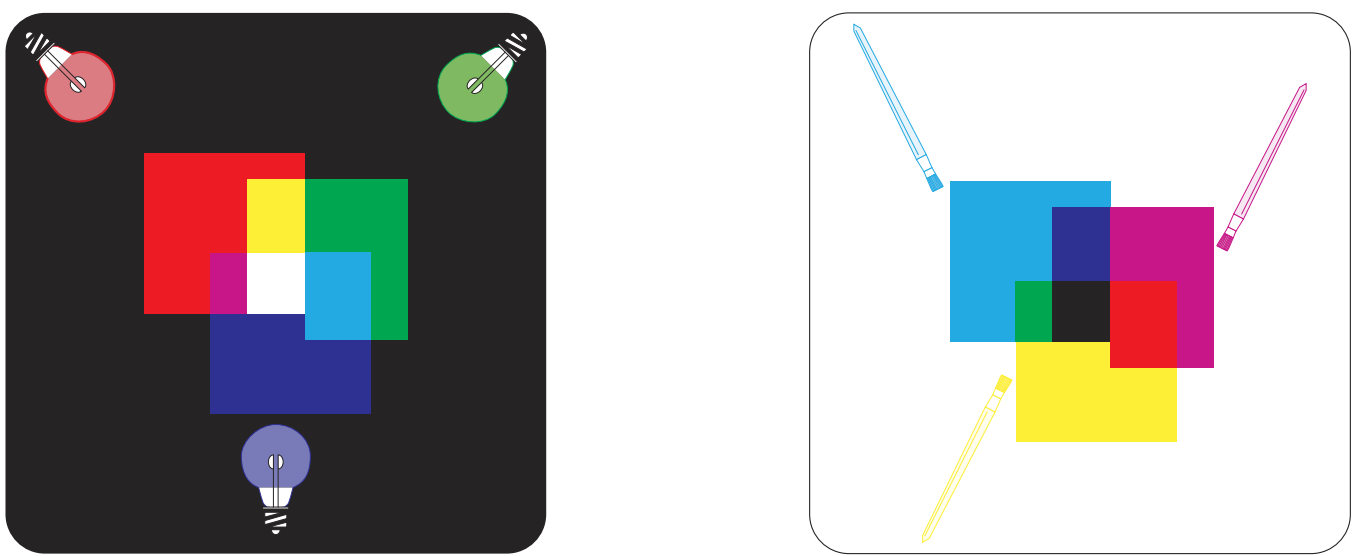

Figure 9. Left: Additive color formation. Right: Subtractive color formation.

As we saw in the color matching experiment, all colors and their combinations in pairs can be elicited by varying the power of the primary light sources. The analogous experiment with the potter's wheel or Alhazen's top is to compose the top's surface with colored sectors of varying angles. The additive combination can also be created by flashing the primary light sources in temporal sequence and phase modulating them for intensity, which is how color matching experiments are carried out in practice with flicker. ${ }^{9}$ Yet another additive color formation consists in using adjacent color dots that are smaller than the eye's resolution, so they are perceived as a monochromatic whole.

The more complex additive color formation techniques are no longer linear, which is especially the case for the adjacent dot technology. When the dots are light emissive, the old cathode ray tube (CRT) technology was linear (up to gamma), but the newer liquid crystal display (LCD) technology is not linear. When the dots are reflective, like in the 1972 Polaroid SX-70 film, the technology is very difficult, as the dots have to be confined in a mosaic or reticle. The early attempts from Lumière's Autochrome to Louis Dufay's Dufaycolor were not successful.

Recently these early additive continuous tone (contone) methods have been extended to bitmap (bi-level) printing on ink-jet printers. Albeit not linear, the colors formed in additive printing can be modeled quite

\footnotetext{
$\S$ Alhazen proposed the scientific process in use today and consisting in observing a phenomenon, formulating a hypothesis, and conducting an experiment to prove it. With this process he discovered that light propagates at very high speed along a straight-line. In his Book of Optics he explains reflection and refraction, the latter as a change in the light's propagation speed, eventually leading to the invention of spectacles.
} 
accurately with Neugebauer's model. The difficulty is that the conventional halftoning methods are not additive and cannot be used. The solution for additive printing is juxtaposed halftoning, but it is computationally prohibitive to model the halftones. In Hersch's halftoning algorithm for additive printing, ${ }^{10}$ a library of all possible cell layouts is compiled and stored (it requires about 100M bytes of memory). During halftoning, the appropriate cells are retrieved and printed.

Despite these heroic efforts over a century, there is a fundamental physiological limitation imposed by the retina. For the system to be linear, the sensitivities of the $S, M$, and $L$ cones would have to be independent, i.e., disjoint or juxtaposed. However, when the sensitivities are measured, the overlapping curves in Fig. 10 are obtained.

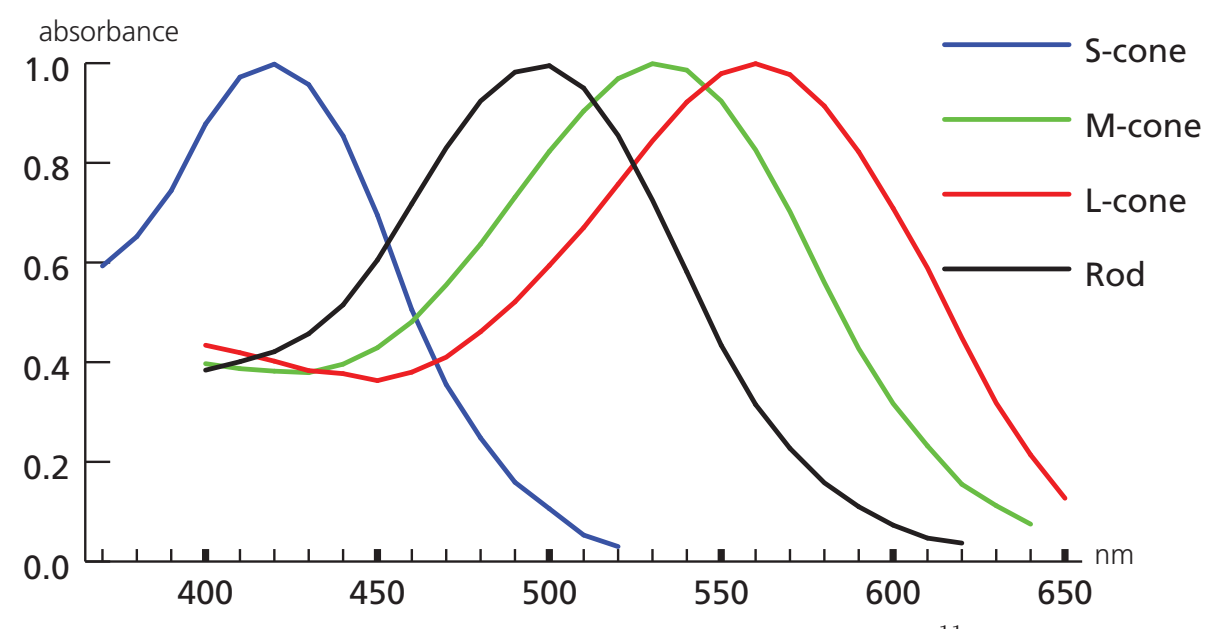

Figure 10. The sensitivities of the human retina. ${ }^{11}$

Therefore, it is not possible to stimulate just one type of cone, there are always unwanted stimulations. In exchange of very dim images, we could use light sources with a very narrow spectrum, but we cannot circumvent the fundamental limitation of not being able to stimulate the cone types independently.

So far we have discussed additive color, where we start from black and then add color. We can also create colors the other way around, starting with a white surface and applying filters to absorb the opposite colors from white to leave a desired color. This is illustrated on the right side of Fig. 9. The white surface can be a sheet of paper, a canvas, or plaster illuminated by a white light. The filters can be inks, paints, colored glass, etc.

In subtractive color formation we not only have the problem of unwanted absorptions, but we additionally have the problem of a wider spectrum (which has the benefit of a brighter image). Figs. 11, 12, and 13 show the spectra of a very high quality printer, using the terms common in the printing industry for denoting the colorants.

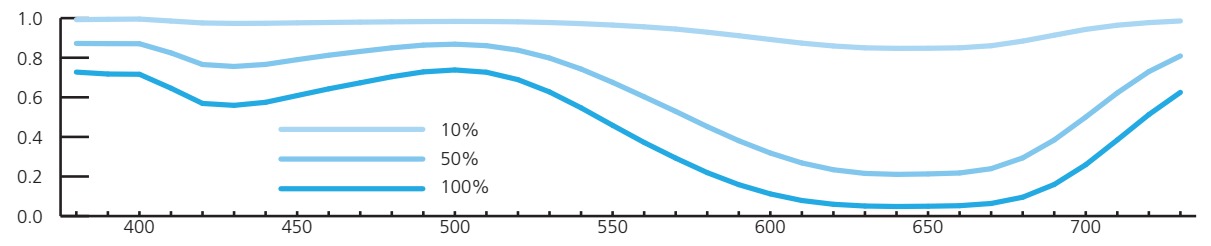

Figure 11. Primary blue, or cyan.

What makes printing with halftones so highly non-linear and complex it that the colors are not created just by superposition of ink filters (subtractive model), but sometimes the primary colorants are printed juxtaposed to each other (additive model). Other times, when printing over an ink that is not yet dry, the inks mix and form a third colorant. 


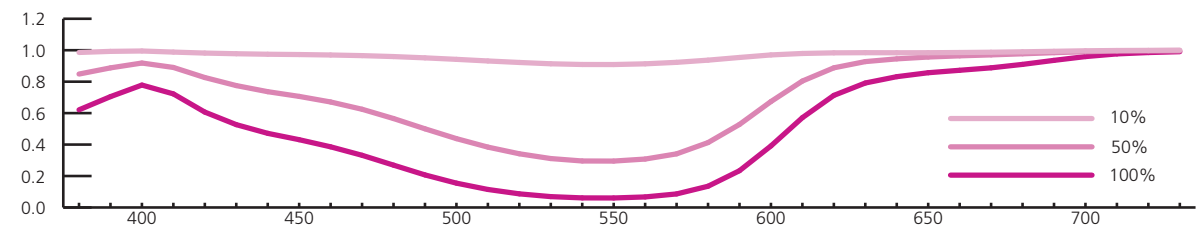

Figure 12. Primary red, or magenta.

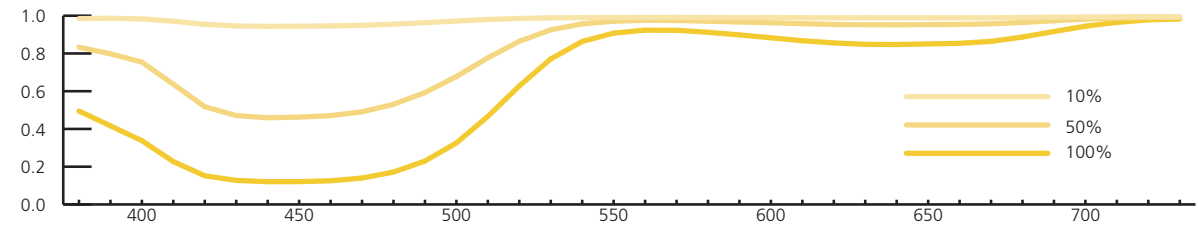

Figure 13. Primary yellow.

These unwanted absorptions are the reason why, for example, it is not possible to build a light lens (analog) color copier. When at PARC Gary Starkweather built Lilac, the first color laser printer/copier, he could do it because it was a digital device built on the scan-print-think principle, where a page is scanned, color-transformed, and then printed. In Lilac, the color transform was a simple matrix, but you could just replace it with the identity matrix to appreciate the huge quality jump achieved by tackling the unwanted absorptions. It may be a sign of corporate myopia that for over a decade after Lilac, product divisions were still trying to develop light lens color copiers.

In the early days, the computing power of embedded devices was relatively low, typically a 6809 or a Z-80. Therefore it was essential to avoid "large swings" by breaking down a printer - be it based on xerography, offset, or gravure technology - into modules and tightly control them through feedback loops, e.g., paper humidity, ink viscosity, blanket tension, temperature everywhere, etc. The reason spectrophotometers like the SPM-700 or an SPM-100 head where embedded, was that measuring and processing a single process gray patch was faster than measuring and processing 4 or 6 inks separately with a scanning densitometer.

The reader at this point might think that color printing is still an immature technology and it is for an insufficient research effort that the inks do not have nice rectangular spectra. In reality, things are not simple and designing inks requires difficult trade-offs. Consider that an object's spectral power distribution is the multiplication of the incident light's spectral power distribution with the object's reflectance curve, as shown in Fig. 14.

In practice, the incident light source is never uniform, but modulated by the ambient through reflections on the face, furniture, plants, etc. If an object's reflectance curve has several local peaks instead of a single narrow peak, there is a more prominent total modulation, which is perceived to be more pleasant (see [12, Section 4]). For this reason a good ink designer will use two pigments, a primary pigment for the dominant hue and a secondary pigment for a subconscious modulation. For example, usually Prussian blue is added to a black colorant.

Another consideration is to reduce metamerism, i.e., we would like an image to appear similarly under different but common light sources, such as sunlight and tungsten light. This robustness for metamerism is achieved by making the ink's reflectance curves broad.

The colorants have also to be durable, i.e., robust for humidity, fading, and gas exposure (mainly ozone and nitrogen oxides). The techniques to prolong print durability influence which dyes and pigments can be used and therefore the reflectance curve.

With all these trade-offs, the the gamut of an ink set must be kept large enough to allow for the reproduction of the most important object colors. In dye based inks, chroma extension is often achieved by adding a second dye admixture. Magenta ink is often produced by adding Color Index Acid Red 52 (a very bright fluorescent blue shade red acid dye) to Color Index Reactive Red 23, cyan by adding Acid Color Index Blue 9 (a brilliant 

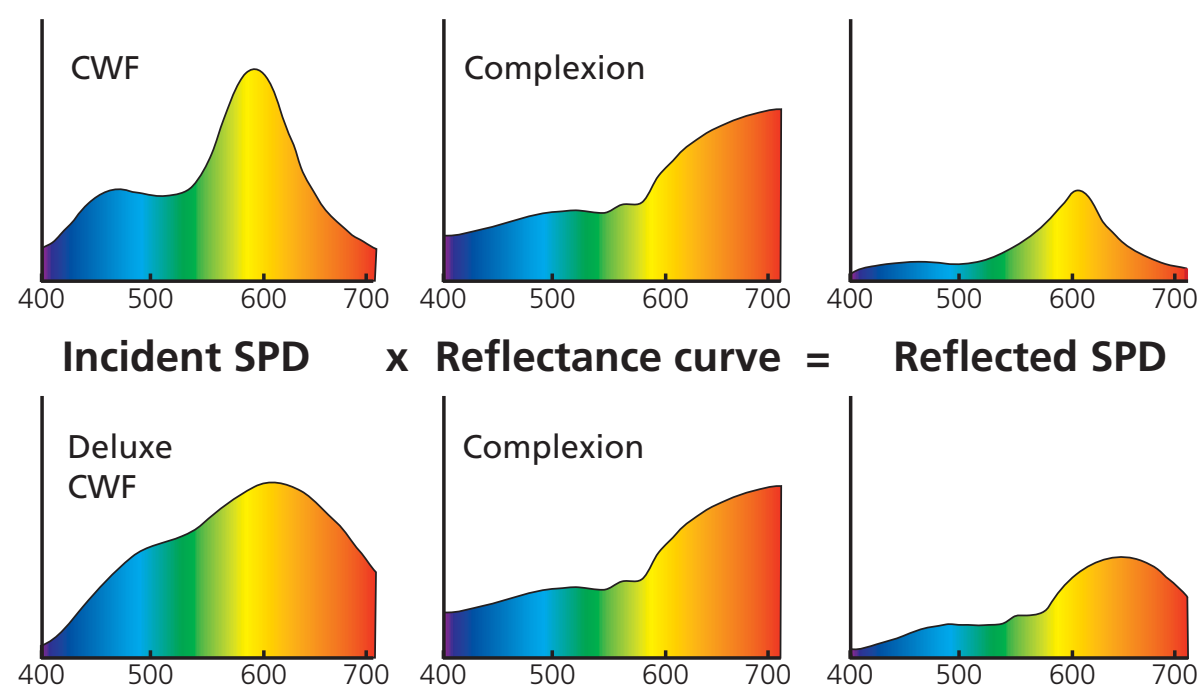

Figure 14. An object's spectral power distribution is the product of the incident spectral power distribution time the spectral reflectance curve.

blue often found in ice cream) to Color Index Direct Blue 199 (the classical turquoise used for writing inks), and similarly for the other colorants. ${ }^{13}$

The conclusion of this digression is that color formation is so complex that we cannot use simple arithmetic formulæ like turquoise + fuchsia $=$ purple to predict the result of mixing colors. The only method for predicting color mixtures is to create tables containing mixture samples in their cells and measuring them. The resulting table giving a trichromatic coordinate for each mixture is then inverted, yielding a table that for a given tristimulus value gives the formula to elicit that color in the viewer.

\section{NAMING COLOR}

To summarize the discussion so far, we have seen the organization of colors, their measurement, and how to reproduce them. We have seen, that we can communicate the appearance of a specific brown, for example, by transmitting three numbers like $(A, T, V)=(20,25,60)$. Although painting by numbers is very efficient, as humans we tend to be not very good at keeping a numerical color atlas in our head, so for our daily life we need a method with a much lower cognitive load.

Language is a set of conventions that allows us to communicate. Since color is an abstract entity, we cannot summon physics to find models that allow us to compute color names. The study of color names is a complex science that is still in its infancy.

First of all, linguists do not speak of color names but of color terms. The basic research technique is to use a table like for the color mixtures. The most used table is compiled from the periphery of the Munsell Color Tree, as shown in Fig. 15.

The researchers studying color terms take this table or chart and approach a group or sub-culture. Each member of this group is then asked to give a term for each color in the table. Finally the responses are averaged over the group members. Such a study was first performed by Berlin and Kay, ${ }^{14}$ but the work has continued through the work of hundreds or thousands of researchers all over the world under the moniker World Color Survey (WCS).

The result of this work is an ontogeny of the color terms, i.e., a hypothesis on how color terms evolved with the evolution of a society, culture, or sub-culture. Usually this ontogeny for all humans is represented in the diagram Fig. 16. The interpretation is that there are only 11 basic or universal color terms. These terms are grouped in 7 stages, and the more a sub-culture is evolved, the more stages it uses. 


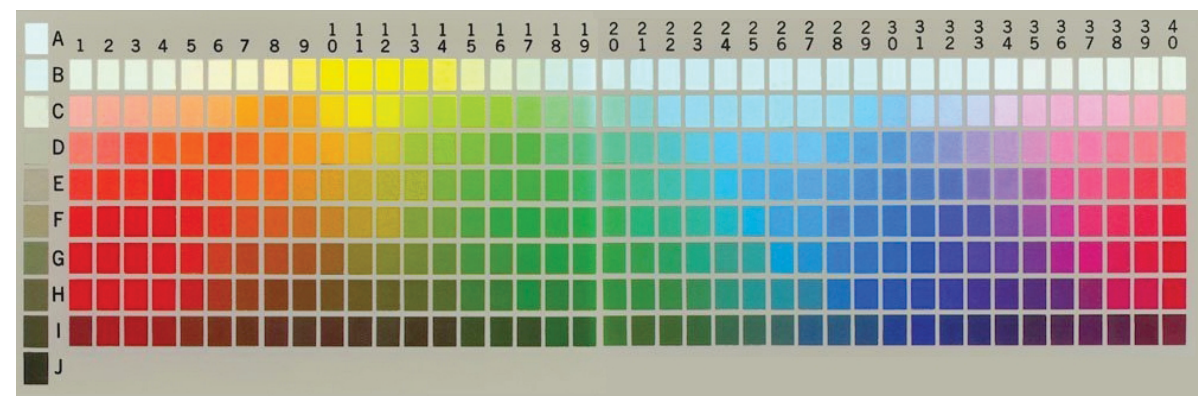

Figure 15. The colors on the surface of the Munsell system color gamut are used in the WCS. The shown colors are very approximative, for experiments you should use either Munsell Sheets of Color or create instances from the specifications in the WCS Data Archives, http://www.icsi.berkeley.edu/wcs/data.html.

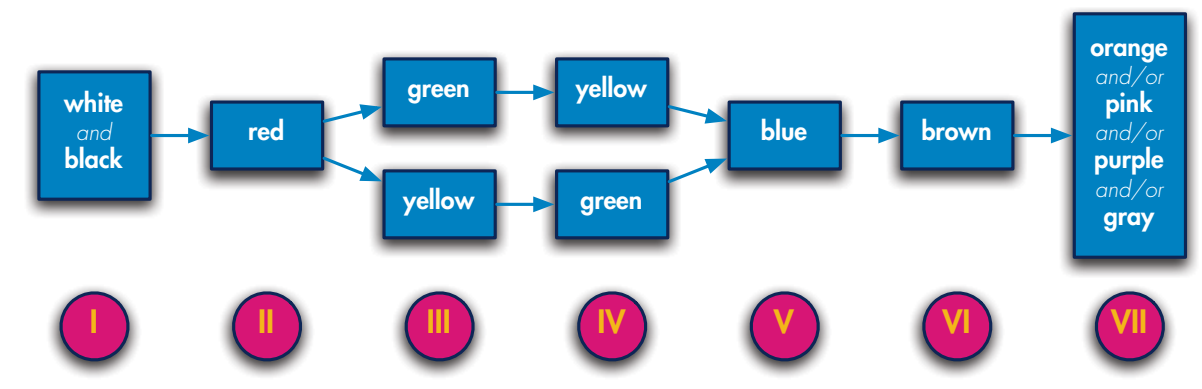

Figure 16. This graph summarizes Berlin and Kay's work, listing the basic color terms and their ontogeny.

One problem with the WCS is that the colors in table Fig. 15 are on the periphery of the Munsell color atlas, so that all muted colors are missing. Boynton and $\mathrm{Olson}^{15}$ performed a strict laboratory experiment in which they used the OSA color atlas mentioned earlier, obtaining the color map shown in Fig. 17.

In the OSA color space, the abscissa $j$ is the amount of yellowness (from the French jaune) and the ordinate $g$ is the amount of greenness. We know that by construction, the OSA atlas is the one that is most uniform. ${ }^{6}$ Yet, note the big variation of the area occupied by the various universal color terms: tiny for grey and red, large for green. Note also that this map has large blank regions for which there are no universal color terms.

There have been several attempts to create a "full" color dictionary, in which there are no blank areas. For

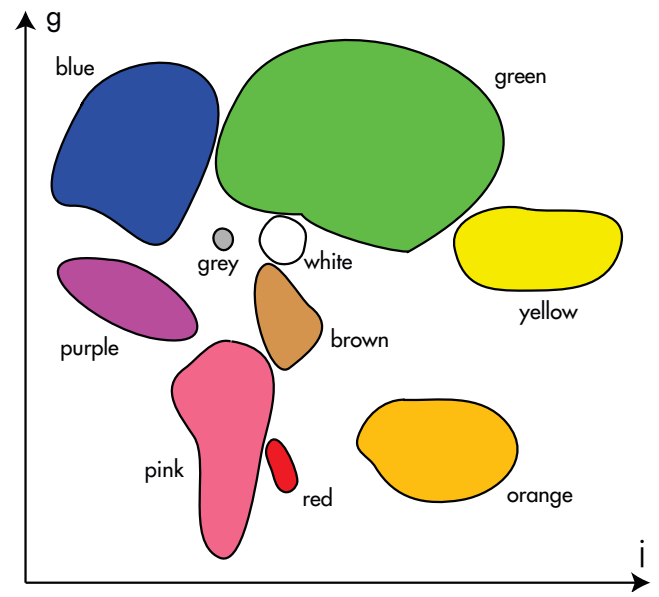

Figure 17. The regions of the universal color terms in the OSA color space. 
example, Nemcsis has assigned terms to all colors in the Coloroid space. ${ }^{16}$ Fig. 18 shows the color terms for the hue sheet 20 shown in Fig. 5.

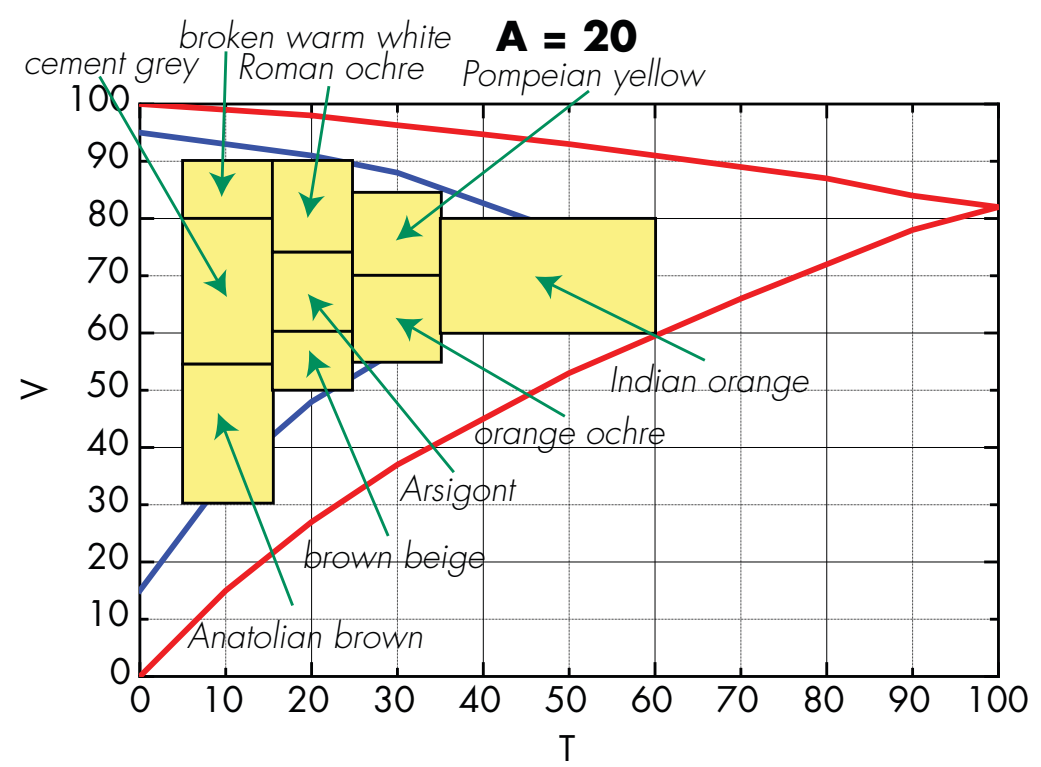

Figure 18. Color terms for the hues in sheet 20 of the Coloroid system (seeFig. 5).

Without looking at Fig. 5, would you be able to visualize Pompeian yellow? Probably an architect working on Budapest's restoration knows exactly what color that is, but for the rest of us this color term remains a mystery. Similarly, to a Palo Altan it may be clear what teal is, because this color was very popular here a decade ago, but it might be less obvious to a Bostonian.

Worse, color terms evolve over time, as is very evident in the Japanese language. ${ }^{17,18}$ In the western culture, purple transitioned from violet to red. According to Zollinger, ${ }^{19}$ from Phœnician times the purple colorant was extracted from molluscs living in the coast of present day Lebanon and Israel. It was a rare colorant, hence used only for textiles destined to the rich and powerful. In fact, in 1464 Pope Paul II decreed that cardinals have to wear purple. By coincidence this was the time the Byzantine empire crumbled and in the Ottoman empire the Purple industry located in the Eastern Mediterranean disappeared. Hence, the tailors had to switch to a red colorant, but the papal decree cast the label purple into concrete. If today you visit a clothing store for clergy, when you ask for purple, you will get a red cape.

One of the color terms that had the most tormented evolution, including turquoise and yellowish, is the Italian term glauco. The Zanichelli vocabulary translates glauco into English as blue-green, sea-green, glaucous. You may be familiar with this color name from glaucoma, a condition of increased pressure within the eyeball, causing gradual loss of sight. Today, in Italian glauco is associated most commonly with eye colors, because of the gray-green haze in the pupil.

To understand how a color term can change its association to apparent color drastically between yellowish and bluish, it is useful to look up the entries around this term in a Classical Greek dictionary:

- glaukiao, to have an inflamed look

- glauk-ommatos, with inflamed eye

- glaukos, scintillating, brilliant, shining || cerulean, azure

- Glaukos, name of many characters

- glaukostes, luster, shine of the eyes \| cerulean color 
- glauko-xros, of azure color, of olive and pale color

- glauk-opis, of the shining, brilliant, cerulean eyes

We can infer that the classical use was for the shining color of eyes, mostly light blue, for example the color of Athene's eyes (glaukopsis). However, in the Homeric epics it has been used also for dark blue. Strangely, glauko-xros has been used also for olive and pale colors.

This strange use might at first explain why then in the later Middle Ages the meaning of the derived Latin term glaucus changed dramatically from blue-gray to yellow. Instead, we would like to speculate here that it might be connected to the loss of diphthongs as Latin became vulgarized. In our Classic Latin vocabulary from middle school we find these two definitions:

- cærúleus, cerulean, azure, turquoise, dark; cærula cæli, or simply cærula, the azure, the azure sky

- cerula, piece of wax

As you see, as the diphthongs were being dropped with the vulgarization of Latin, cerulean blue might have become the color of wax. Indeed, Roger Bacon $(\sim 1214-1292)$ used glaucitas to refer to yellowness and ceruleus (wax color) as a yellow color falling between glaucus and citrinus (yellow and orange, respectively). In the 14th century, Theodoric of Freiburg used glaucus with the same meaning. But then, in the mid-16th century, Scaliger (1557) put it back in the blue category, where it still was in the beginning of the 17th century with Francois d'Aguilon [2, p. 8].

If the term purple changed as a consequence of the collapse of a major civilization, the term glaucus might have been done in by an overworked or sloppy monk. In this case, glauko-xros used for olive and pale colors could be a medieval mistake rather than a genuine classical Greek color term.

Another tormented color term is fuchsia, whose claim to fame is to be the most misspelled color term in our online color naming experiment. The name fuchsia comes from a flowering plant that in the western culture was identified in the late 17th century by Charles Plumier, who in 1703 named it after the German botanist Leonhart Fuchs $(1501-1566)$. More precisely, the color term comes from the colorant fuchsine, first made from coal tar dyes in the year 1859 .

In color naming experiments fuchsia is different but close to magenta. Legend has it the name comes from the City of Magenta, where 152 years ago a battle took place that left the fields in the magenta color from the soldier's blood. But the magenta color is not the color of Magenta, because in Italian by color of a city we mean the colors in its crest. Therefore, the colors of Magenta are yellow and black.

The little village of Magenta near Épernay, smack in the middle of Champagne, got its name from the Italian Magenta 152 years ago, to celebrate the French victory over the Austrians in the battle of Magenta. Unlike the yellow and black original Magenta, the French Magenta is all magenta, starting from its crest. Their magenta Web site explains that in 1859, a new red colorant with a pink slant had been discovered, and to commemorate the French blood spilled in the battle of Magenta, it was renamed from fuchsine to magenta (and not because of the fields of Magenta being red from blood). In 1860 this color became fashionable in London as red magenta.

Therefore, fuchsia and magenta are true synonyms by etymology. Yet, on average, we use these terms to designate different colors. The perceptual distance between fuchsia and magenta can thus be used as an indication of precision in color naming.

Given this ephemerality of color terms, how can we find terms for all colors we need for a color theory? Our solution is the use of crowd-sourcing, as we detailed in last year's paper at this conference ${ }^{20}$ and summarized in the flowchart Fig. 19.

The color naming tool gives us terms for the colors, and we now know that we cannot use the terms for doing color name arithmetics to predict color mixtures: both expression in the title of this paper are wrong! The question is whether we can do something in between, like expressing color theories through color terms, because this would be very useful for didactic purposes. 


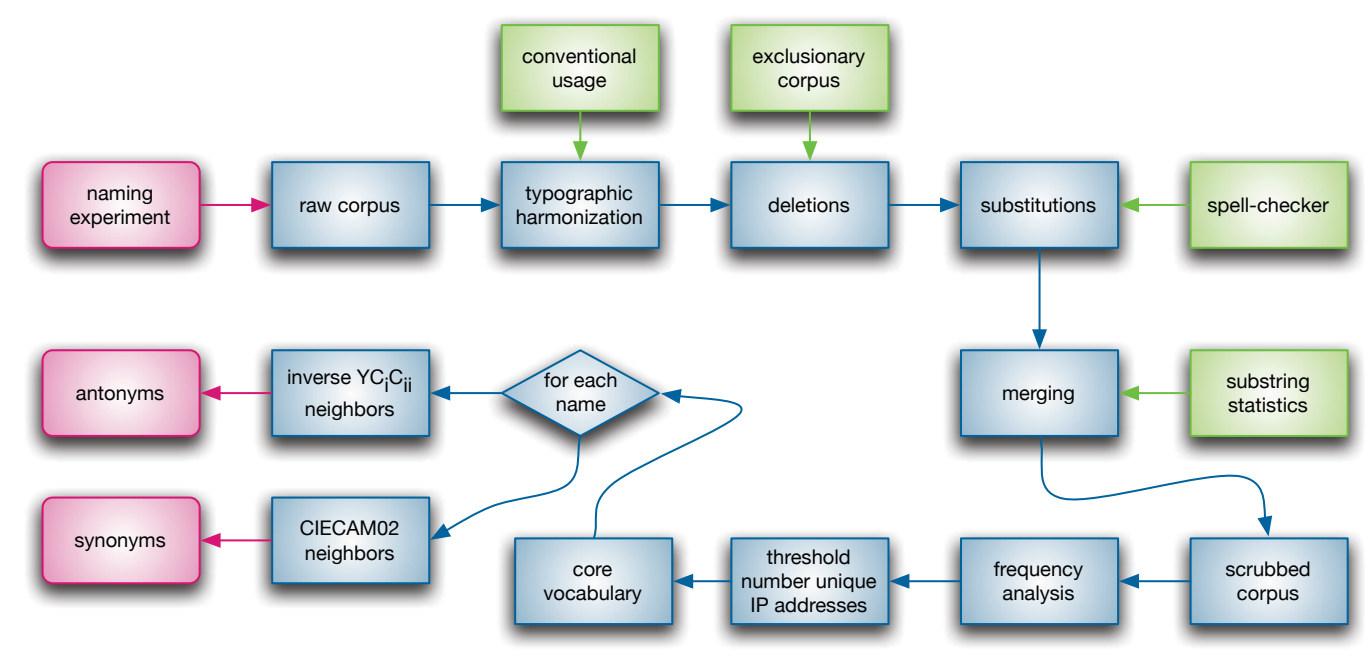

Figure 19. Workflow for harvesting color terms though crowd-sourcing. ${ }^{20}$

If the color space used for the color theory is perceptual, there are some properties we can express algorithmically, for example the complement of a color can be calculated in the underlying color space by considering the antipode. ${ }^{21}$ However, compare the right side of Figs. 3 and 4: in Leonardo's model the complement of blue is yellow, but in Itten's model it is orange.

If you do a survey asking people to name the complements of colors, you may get still a different answer, due to the intrusion of color semiotics. Respondents might associate blue with the symbol for cold, whose antonym is hot with color symbol red. Therefore, the semiotic complement of blue could well be red. At this point in time, determining the antonyms of color terms is still an open research problem. Actually, it might not even be clear what is the difference between antonym, opponent, and complement.

\section{VALIDATING CROWD-SOURCING}

For now, we are not able to tell whether blue's complement is yellow, orange, or red. However, we have made progress in validating crowd-sourcing through a large controlled experiment. Leveraging on all the good research on the universal color terms, we can focus on the non-basic color terms.

At HP Labs, we have periodic lunch socials at work and each social event is hosted by several of the labs. Our lab was to be one of the host labs for an upcoming lunch and during the planning phase this basic idea was formulated: what if we gave our co-workers randomly colored patches and had the dining tables labeled with color terms - how would people select their dining tables?

To start with, 25 non-basic color terms were selected from our color thesaurus and pseudo-random noise was added to the corresponding centroids. This is harder than it sounds. The terms purposely excluded the basic WCS color terms of red, green, blue, yellow, black, white, gray, purple, pink and brown. Otherwise, the game would be too easy. The non-basic terms were selected to both cover a wide range in color space and to have some overlap between the dining tables or color samplings. The centroids were taken from the color thesaurus. ${ }^{20}$ A visualization of the original color chips is shown in Fig. 20.

Next, the colored patches were assigned a unique psuedo-random identifier and printed on high gloss media with a calibrated ink-jet printer. The spectral reflectance curves were then measured for each of these patches. A preliminary calculation of the mean color difference from the mean in CIE $1976\left(L^{*} a^{*} b^{*}\right)$ was used to determine that the above sampling had roughly equivalent variation per centroid. Caveats apply, but the point is that each patch was measured and with a measurement of the illumination conditions of the cafeteria the calibration of the lunch social event is complete.

After the patches were printed and measured, the physical dining tables were arranged. We set up 25 tables of 10 chairs each, in a radial configuration. Each table had a balloon with a non-basic color term according to 


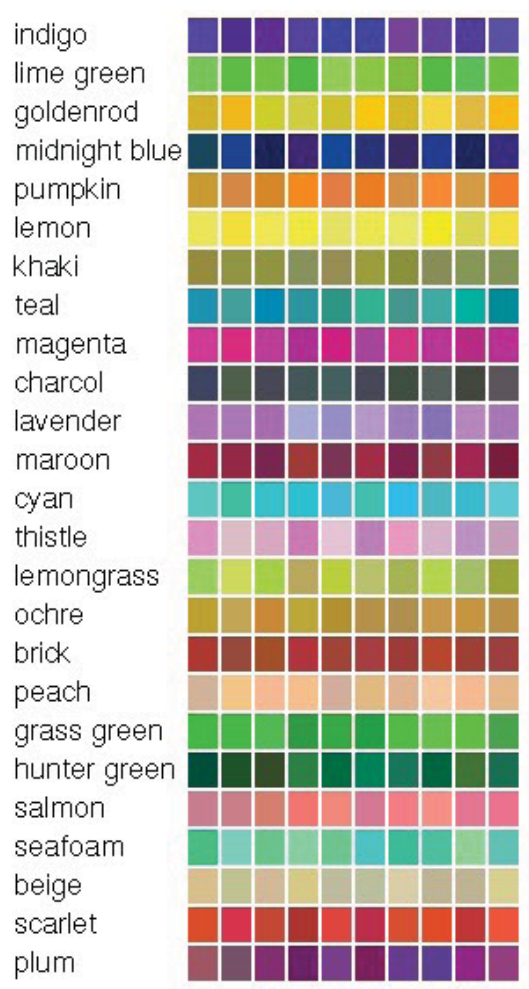

Figure 20. Color chips given to diners upon entering the cafeteria (multiple copies were made). The color terms are from the color thesaurus ${ }^{20}$ and the chip colors were created by adding noise to the centroids.

Fig. 20 on it. Printed instructions were placed on each table and the instructions were also given verbally while people waited in line for food. The instructions were as follows:

- Please take a colored patch from one of the volunteers before getting your food

- The game consists of sitting at the table with color name closest to your colored chip

- If you have any difficulties with selecting a table please ask for help from anyone

- The table with closest agreement or smallest difference between their color patches will win a prize

- One member of the winning table will then be eligible to win the grand prize

Note that this was in fact a competitive calibrated lunch. At this point we will skip over the observed individual and group dynamics of the dining table selection and optimization. This is arguably a key part of this experiment, but most likely insights gained here will show up in future papers and analysis. Suffice to say, people seemed to be enjoying themselves.

Now on to the results. A computational test was done to remove obvious outliers (such as the two pumpkins that sat at the teal table). Interestingly the number of outliers was quite low, around $4 \%$ of the participants. After outlier removal, the final color seating arrangement was as shown in Fig. 21.

After some weighted and non-weighted calculations of the mean color difference from the mean in CIE 1976 $\left(L^{*} a^{*} b^{*}\right)$, the lime green and thistle tables were announced as winners. Hooray for lime green and thistle!

So how do the results for the calibrated lunch compare to the uncalibrated web?

The CIECAM02 hue angles for the non-basic color terms were computed for both the final configuration of measured printed color patches for the calibrated lunch and plotted versus the corresponding uncalibrated, but 


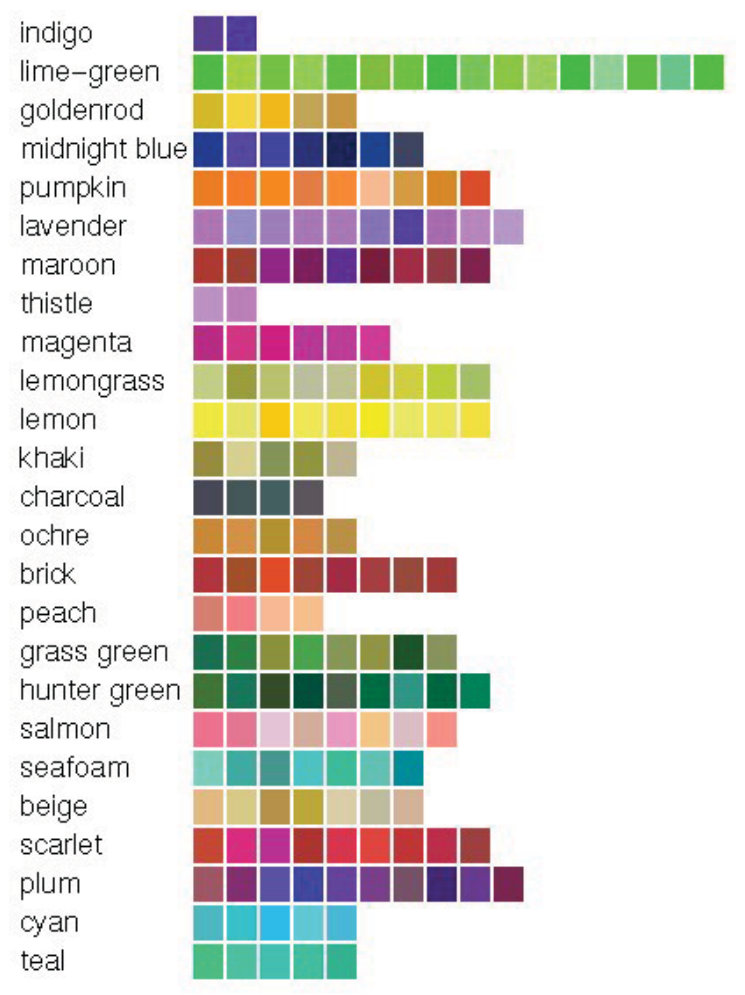

Figure 21. Color seating arrangement chosen by the members of HP Labs.

assumed to be sRGB, Web color centroids taken from the online color naming experiment. The results are shown in Fig. 22 and $R^{2}=0.99$.

Unresolved issues to be addressed in a future experiment include using a more uniform color space than CIE $1976\left(L^{*} a^{*} b^{*}\right)$, with a better difference metric, and using non-parametric statistics for the expected value.

\section{CONCLUSIONS}

When communicating color, it is convenient to use terms (color names). However, these terms cannot be used to predict color mixtures with simple arithmetic formulæ. Although some color theory operators can be expressed with color terms, the results depend on the underlying color space and its construction.

We have developed a detailed derivation and explanation of how the various concepts and methods are related. We hope this can be of benefit to those teaching color.

Given the ephemerality of color terms, one of the most challenging problems is to compile color thesauri. In previous papers we have proposed crowd-sourcing as an efficient experimental technique. Due to the uncontrolled conditions, this technique is often contested, although highly esteemed experts have endorsed it. ${ }^{22}$ We have described a large controlled experiment confirming our early data obtained though crowd-sourcing.

\section{ACKNOWLEDGMENTS}

We are indebted to Renata Pompas and Lia Luzzato for starting the conversation on teaching color theories through arithmetics with color terms; this paper's title was one of their questions. Prithviraj Banerjee, Miheer Bhachech, Doris Chun, Robert L. Cobene II, Gary J. Dispoto, Richard E. Elder, Nina Guzman, Dick Henze, Eric Hoarau, Bill Holland, Kok-Wei Koh, Norma Lerma, Paul F. Matheson, Laurie S. Mittelstadt, Ido Ofir, John Ludd Recker, and Wenjia Zhang assisted in the large controlled experiment. 


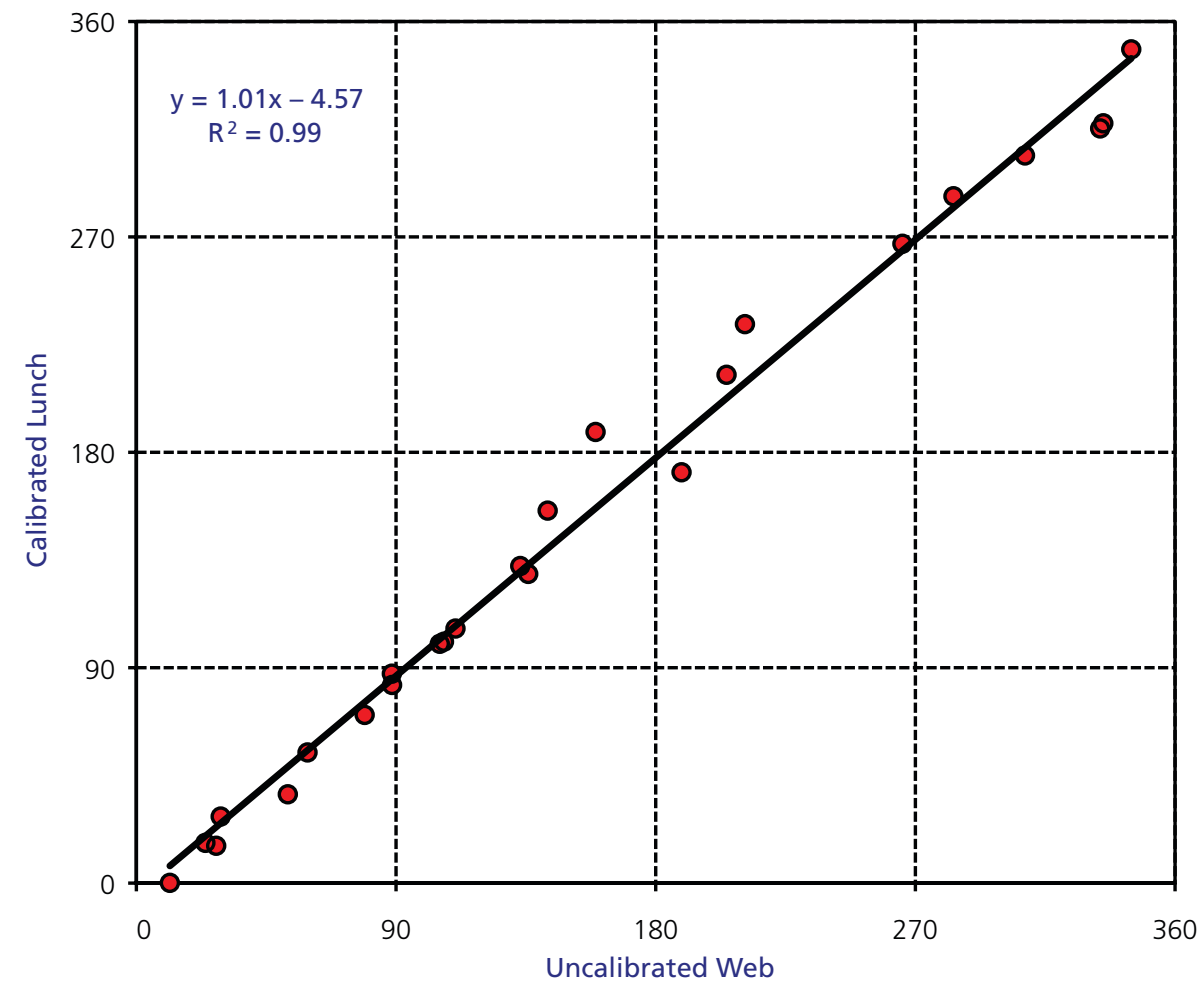

Figure 22. CIECAM02 hue angles for 25 non-basic color terms. Calibrated lunch vs. Web color centroids taken from the online color naming experiment though crowd-sourcing. $R^{2}=0.99$ indicates that crowd-sourcing is a very reliable technique for research in color science.

\section{REFERENCES}

[1] Vanhaeren, M., d'Errico, F., Stringer, C., James, S. L., Todd, J. A., and Mienis, H. K., "Middle Paleolithic Shell Beads in Israel and Algeria," Science 312(5781), 1785-1788 (2006). http://www.sciencemag.org/ cgi/content/abstract/312/5781/1785.

[2] Kuehni, R. G. and Schwarz, A., [Color Ordered - A Survey of Color Systems from Antiquity to the Present], Oxford University Press, New York, NY, USA (December 2007).

[3] da Vinci, L., [Trattato della Pittura], Langlois, Paris, 2nd ed. (1701).

[4] Itten, J., [Kunst der Farbe (Studienausgabe)], Ravensburger Buchverlag Otto Maier GmbH (1987).

[5] Beretta, G. B., "Color palette selection tools," in [Advanced Printing of Conference Summaries, SPSE's 43rd Annual Conference], 94-96, The Society for Imaging Science and Technology (May 20-25 1990).

[6] Nickerson, D., "OSA uniform color scale samples: A unique set," Color Research \&3 Application 6, 7-33 (Spring 1981). http://onlinelibrary.wiley.com/doi/10.1002/col.5080060105/abstract.

[7] Nemcsics, A., [Coloroid Colour Atlas], Innofinance, Budapest (1985).

[8] Kuehni, R. G., "A brief history of disk color mixture," Color Research E3 Application 35(2), 110-121 (2010). http://onlinelibrary.wiley.com/doi/10.1002/col.20566/abstract.

[9] Wyszecki, G. and Stiles, W., [Color Science: Concepts and Methods, Quantitative Data and Formulce], John Wiley \& Sons, New York, 2nd ed. (1982).

[10] Hersch, R. D., Donzé, P., and Chosson, S., "Color images visible under UV light," ACM Trans. Graph. 26(3), 75 (2007). http://doi.acm.org/10.1145/1276377.1276471.

[11] Dartnall, H., Bowmaker, J., and Mollon, J., "Human visual pigments: Microspectrophotometric results from the eyes of seven persons," Royal Society of London Proceedings Series B 220, 115-130 (November 1983). http://rspb.royalsocietypublishing.org/content/220/1218/115. abstract. 
[12] Beretta, G. B. and Nonaka, Y., "House painting with NCS in the USA," Atti della Fondazione Giorgio Ronchi LV(1), 93-118 (2000).

[13] Kazlauciunas, A., "Photo-realistic ink jet digital printing — factors influencing image quality, image stability and print durability," Coloration Technology 126, 315-324 (December 2010). http://dx.doi .org/10.1111/ j.1478-4408.2010.00271.x.

[14] Berlin, B. and Kay, P., [Basic Color Terms: Their Universality and Evolution], University of California Press, Berkeley (1969).

[15] Boynton, R. M. and Olson, C. X., "Locating basic colors in the OSA space," Color Research 83 Application 12, 94-105 (April 1987). http://onlinelibrary.wiley.com/doi/10.1002/col.5080120209/abstract.

[16] Nemcsics, A., [Colour Dynamics: Environmental Colour Design], Akadémiai Kiadó, Budapest (1993).

[17] Stanlaw, J., "Two observations on culture contact and the Japanese color nomenclature system," in [Color categories in thought and language], Hardin, C. L. and Maffi, L., eds., ch. 11, 240-260, Cambridge University Press (1997).

[18] Tominaga, S., Ono, A., and Horiuchi, T., "Investigation and analysis of color terms in modern Japanese," Color Imaging XV: Displaying, Processing, Hardcopy, and Applications 7528(1), 752804, SPIE (2010). http://dx.doi.org/10.1117/12.838884.

[19] Zollinger, H., [Color: A Multidisciplinary Approach], Helvetica Chimica Acta, Zürich (1999).

[20] Beretta, G. B. and Moroney, N. M., "Color naming: color scientists do it between Munsell Sheets of Color," Color Imaging XV: Displaying, Processing, Hardcopy, and Applications 7528(1), 75280V, SPIE (2010). http://dx.doi.org/10.1117/12.846957.

[21] Beretta, G. B., "Functional color selection system." U.S. Patent 5,311,212 (May 10 1994).

[22] Boynton, R. M., "Insights gained from naming the OSA colors," in [Color categories in thought and language], Hardin, C. L. and Maffi, L., eds., ch. 6, 135-150, Cambridge University Press (1997). 